\title{
Mathematical Programming Model for the Two-Level Facility Location Problem: The Case of Tanzanian Emergence Maize Distribution Network for 2004-2010 Maize Data
}

\author{
Said A. Sima \\ Department of Mathematics, University of Dar es Salaam, Box 35062, Dar es Salaam, Tanzania \\ E-mail: said.sima@gmail.com
}

Received 18 Jun 2020, Revised 14 Jul 2021, Accepted 20 Jul 2021, Published Aug 2021

DOI: https://dx.doi.org/10.4314/tjs.v47i3.13

\begin{abstract}
A two-level facility location problem (FLP) has been studied in the transportation network of emergence maize crop in Tanzania. The facility location problem is defined as the optimal location of facilities or resources so as to minimize costs in terms of money, time, distance and risks with the relation to supply and demand points. Distribution network design problems consist of determining the best way to transfer goods from the supply to the demand points by choosing the structure of the network such that the overall cost is minimized. The three layers, namely production centres (PCs), distribution centres (DCs) and customer points (CPs) are considered in the two-level FLP. The flow of maize from PCs to CPs through DCs is designed at a minimum cost under deterministic mathematical programming model. The four decisions to be made simultaneously are: to determine the locations of DCs (including number of DCs), allocation of CPs to the selected DCs, allocation of selected DCs to PCs, and to determine the amount of maize crop transported from PCs to DCs and then from DCs to CPs. The modelled problem generated results through optimization with respect to optimal location-allocation strategies. The results of the optimized network shows the improvement in costs saving compared to the manually operated existing network. The results show the costs saving of up to $18 \%$ which is equivalent to $\$ 2,910$ thousand (TZS 2.9 billion).
\end{abstract}

Keywords: Optimization, Maize crop, Transportation network, Deterministic model, Facility location.

\section{Introduction}

Distribution network design problems consist of determining the best way to transfer goods from the supply to the demand points by choosing the structure of the network such that the overall costs are minimized (Ambrosino and Scutella 2005). Here, the network is considered from a graph theory point of view where a connected graph has sets of vertices (nodes) and edges (arcs). In this context, production centres, warehouses (distribution centres) and customer points/demand points are assumed to be vertices, while edges or arcs can act as roads and/or railways or other pathways such as marine routes or flight routes.

In the facility location problem (FLP), it is required to determine the optimal location of facilities or resources so as to minimize costs in terms of money, time, distance and risks in relation to supply and demand points (Ahuja et al 1993, Nagy and Salhi 2007). Sajjadi defined FLP as follows: - "given a set of facility locations and a set of customers who are served from the facilities, then which facilities should be used? Which customers should be served from which facility so as to minimize total cost 1020 
of serving all customers?" (Sajjadi 2008). Some examples of such facilities are schools, warehouses, hospitals, markets, industries, stadium or open space, terminal bus stand (hub), railway stations, military centres, post offices, fire stations and worship places (Cox 1998, Klose and Drexl 2005, Sajjadi 2008).

The FLP is a broad study area within the location analysis, where the location, allocation and shipment or transportation decisions are solved simultaneously. Usually, the allocation of customers to a specific facility has implication to transportation of goods from that facility to the respective customers. In this context, each customer is supplied directly from a facility without depending on other customer's demands (Klose and Drexl 2005, Ameli et al 2009, Melo et al 2009). The FLP is said to be a two-level problem when there are two transportation levels; from PCs to DCs and from DCs to CPs.

This work studied a two-level FLP so as to optimize the costs of the network which is important for food security at the customers' demand locations. The motivation for this study is fact that the existing literatures in FLP do not address the maize crop distribution network in Tanzania (Snyder 2006, Nagy and Salhi 2007) to the best of knowledge. The objective is to come up with food distribution system that is economical and cost effective for emergence maize distribution in Tanzania. The network is useful during an emergency situation to rescue areas with food shortage. The main task is to analyse the existing distribution set-up in order to study whether it is optimal in terms of cost. This network has five existing DCs where it is possible to vary their capacities during optimization. The flow of maize from PCs to CPs through DCs is optimized. There are two tasks in the analysis of the existing network: Firstly, the optimization of the flow of maize in the existing distribution network. Through optimization of the model, the optimal solution obtained will be compared to the cost of manually operated network. In this case, capacities of DCs will be considered as constant terms. Secondly, the improvement of the existing network through optimization tools is studied. The aim is to satisfy the customers' demand while minimizing the overall network cost. The same five DCs are used but with variable capacities in order to find the best capacities with minimum cost. The capacities in this case are considered as decision variables.

The results from optimization of the existing network will help to recommend cost reduction measures to the Tanzanian government with regard to emergency maize distribution. The main objective is to determine whether to keep the current maize distribution network or to use a different network. Scientific methods, particularly optimization results are used to give an answer to the raised question. The problem is modelled and solved with respect to optimal location-allocation strategies. Specific objectives are as follows:

- To develop a deterministic mathematical programming model for two-level FLP;

- To apply the developed model to Tanzanian emergence maize distribution network;

- To analyse and compare the results obtained.

This study is useful as it will provide a mechanism for reducing food prices within the country during disasters.

\section{A two-level facility location problem for maize distribution network in Tanzania}

The Ministry of Agriculture, Food Security and Cooperatives (MAFSC) of Tanzania, is responsible for food security. The major cereal crops produced in the country are maize (corn), rice (paddy), millet, finger-millet, sorghum and wheat. The country's major food crops (main staple crops) are maize and rice. The problem considered in this paper is maize production and its distribution system in Tanzania. This research considered only maize distribution as per available data, and also maize is the only food crop which is stored in the DCs and is managed by the National Food Reserve Agency (NFRA) under the MAFSC for emergency situations (URT MAFSC 2013). 
The emergency situations considered are acute food shortage in some places in the country (due to drought and other disasters). The stored crop helps in price stabilization in the markets, especially in the urban areas. In the country, there are some common deficit zones due to drought and other weather effects like shortage of rainfall in semi-arid areas.

The food crops production in the country is highly concentrated in the southern highlands regions (Rukwa, Katavi, Songwe, Njombe, Mbeya, Iringa, Morogoro and Ruvuma) and the peripheral areas of the country as shown in Figure 1. On the other hand, the traditional food deficit areas are located mostly in the central corridor regions (Singida, Dodoma and Tabora) and northern parts (Arusha, Manyara, Kilimanjaro and Tanga), and other parts as shown in the map of Tanzania (Figure 1). The specific locations of existing DCs (warehouses) are also shown in Figure 1. The DC in this context is a storage building where commodities are stored for some time before being taken to customers. The specific demand points (customer points); are not shown in the map for clarity; rather some major demand zones have been marked, but the production centres are within the marked production zones, particularly in the southern highlands.

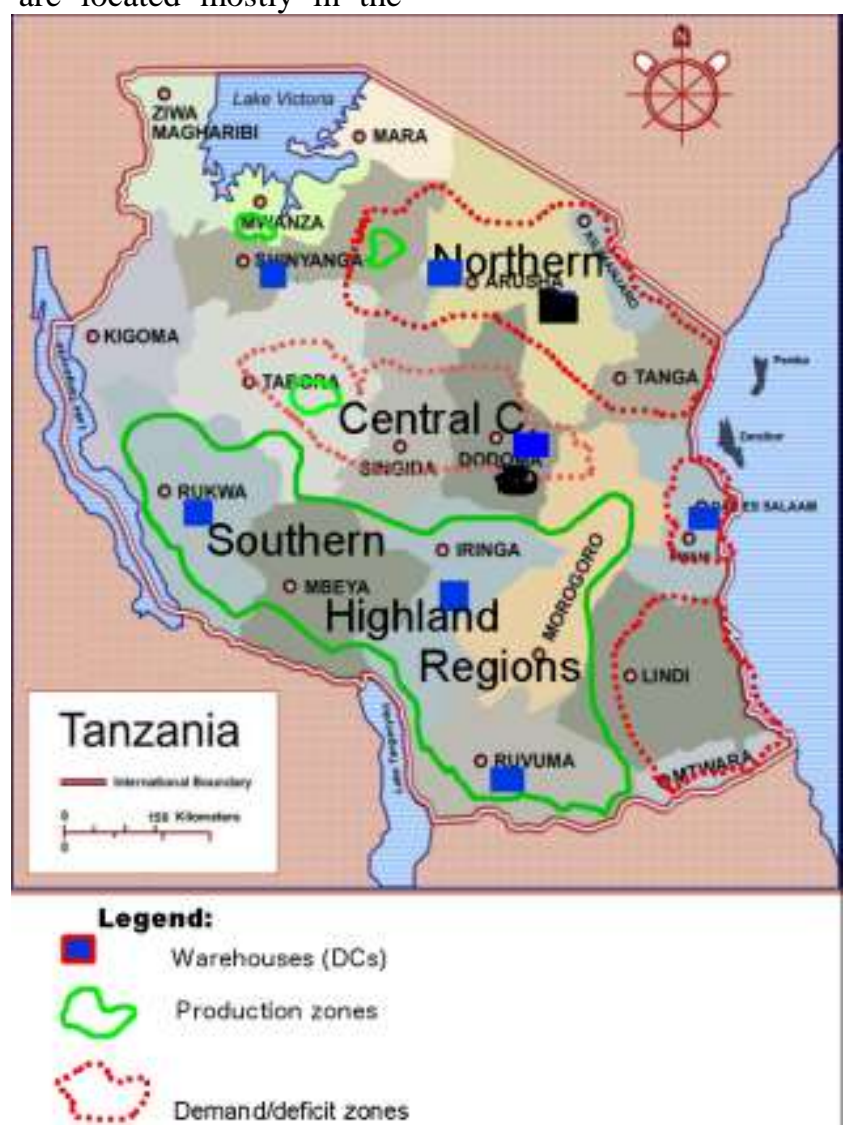

Figure 1: The map of Tanzania showing the food production zones, DCs and demand zones.

Major maize surplus production is from the four regions (known as 'The Big four') namely, Rukwa, Mbeya, Iringa and Ruvuma (Mkenda and Campenhout, 2011). However, these regions had been subdivided into other new regions that include Njombe, Songwe and 
Katavi. This study considers original four regions to concur with the collected data. The specific PCs form a production zone in the southern highlands part of the country. In this study, the PCs will form the first layer among the three layers in the two-level FLP. The maize crops are bought from this production zone by the NFRA for storage in the DCs.

The DCs are scattered in different parts of the country. Usually the storage in the DCs is done for a year (a harvest season to the next harvest season). There are seven existing DCs with a total capacity of 241 thousand tons. These are Arusha (39 thousand tons capacity), Dar es Salaam (52 thousand tons), Dodoma (39 thousand tons), Shinyanga (14.5 thousand tons), Makambako-Iringa (34 thousand tons), Songea (24 thousand tons) and Sumbawanga (38.5 thousand tons). These DCs, as shown in Figure 1, form the second layer of the twolevel FLP model of the study. The first five DCs are used for storage of maize to be supplied to the deficit CPs throughout the country. The last two DCs, Sumbawanga and Songea, are used as reserve DCs to buffer the other five DCs. These two DCs are located in the production zones. The third layer of the model in this study is CPs. These are specific demand points in the country to be supplied by DCs during food deficit time. As indicated in Figure 1, the major deficit zones are central corridor zone and the northern zone. The three layers form the distribution system that needs to be designed at minimum cost while satisfying the customers' demands.
In Tanzania, physical access to food is affected by inadequate transportation infrastructure. Due to long distances between the food PCs, DCs and CPs, together with inadequate and unreliable transportation network to some places, high transportation costs are among the challenges. For instance, the existing distribution system has distances ranging from120 kilometres $(\mathrm{km})$ to $1,348 \mathrm{~km}$ between PCs and DCs. The distances between DCs and CPs, on average, also range from 136 $\mathrm{km}$ to $360 \mathrm{~km}$. This results, at times, to high food prices in deficit areas, and therefore affects access to food by both low incomes, rural and urban populations (Mkenda and Campenhout 2011, USAID 2011). Table 1 gives the summary of PCs, DCs and CPs; and their location zones within the country. The southern highlands zone is the only zone with PCs and also 3 DCs out of the 7 DCs. Notably, based on 2010 data, the Ruvuma, Rukwa, Kigoma and the Dar es Salaam regions have no CPs. All the mentioned regions except Dar es Salaam are self-sufficient in cereal crops production and the surplus production is always expected. The Dar es Salaam region is the place of city dwellers that is populated mostly by employed people who earn salaries. Food deficiencies are mostly realized by people living in the rural areas. The list of DCs and their respective CPs of the collected actual distribution data from 2004 to 2010 were obtained from NFRA.

Table 1: PCs, DCs and CPs distribution in the country (Source: NFRA, Prime Minister Office)

\begin{tabular}{lllll}
\hline Zone & Specific Regions & \# of PCs & \# of DCs & \# of CPs \\
\hline Southern Highlands & Iringa, Rukwa, Mbeya, Ruvuma & 4 & 3 & 9 \\
Central Corridor & Dodoma, Singida,Tabora & 0 & 1 & 17 \\
Northen & $\begin{array}{l}\text { Arusha, Manyara, Tanga, } \\
\text { Kilimanjaro }\end{array}$ & 0 & 1 & 24 \\
Southern Corridor & Mtwara, Lindi & 0 & 0 & 8 \\
Eastern & Dar es Salaam, Coast, Morogoro & 0 & 1 & 11 \\
Lake Victoria & Shinyanga, Mwanza & 0 & 1 & 24 \\
& Mara, Kagera, Kigoma & & & \\
\hline & Total & $\mathbf{4}$ & $\mathbf{7}$ & $\mathbf{9 3}$ \\
\hline
\end{tabular}




\section{Materials and Methods}

Mathematical programming model for the two-level facility location problem

This section presents the deterministic mathematical model of two-level FLP. Here, a deterministic model with a single-product and a single-period planning horizon is considered. The aim is to design a deterministic capacitated two-level FLP and to optimize location, allocation and hence transportation decisions for the distribution network. The deterministic mathematical model for a single period demand consists of four months which are from January to April in each year. The model is adapted from Elhedhli and Goffin (2005) and other references (Azad and Davoudpour 2013, Hindi et al. 1998, Klose and Drexl 2005, Lashine et al 2006, Sahin and Sural 2007).

The notations used in the model are as follows: $\mathrm{J}$ : is the index set for PCs, where $\mathrm{j} \in \mathrm{J}$ and $|\mathrm{J}|$ denotes the total number of PCs, i.e., $P C_{j}, j \in J$ . $P C_{j}=\mathrm{PC}$ allocated at site $\mathrm{j}$.

$\mathrm{K}$ : is the index set for DCs, where $\mathrm{k} \in \mathrm{K}$ and $|\mathrm{K}|$ denotes the total number of possible DC sites, i.e., $D C_{k}=\mathrm{DC}$ located at site $\mathrm{k}, \mathrm{k} \in \mathrm{K}$.

$\mathrm{L}$ : is the index set for CPs, where $1 \in \mathrm{L}$ and $|\mathrm{L}|$ denotes the total number of CPs, i.e., $C P_{l}$,

$1 \in \mathrm{L} . C P_{l}=\mathrm{CP}$ located at site $1,1 \in \mathrm{L} . C P_{l}$ has fixed location together with their associated demand, $D_{l}$.

$R_{k}$ : the set of capacities of given $D C_{k}$. Hence $R_{k}=\left\{V_{k}^{1}, V_{k}^{2}, \ldots, V_{k}^{\left|R_{k}\right|}\right\} \quad$ where $V_{k}^{\left|R_{k}\right|}$ is possible set of capacities for given $D C_{k} \cdot V_{k}^{r}$ is possible set of capacities of given $D C_{k}$.

$S_{j}$ : Supply (production capacity) of a maize crop at $P C_{j}$.

$D_{l}$ : Total demand for four months for maize crop at $C P_{l}$ transported only once in a week. This amount was considered to be transported in the first week of the four months period (January to April) of a year.

$F_{k}^{r}$ : Total fixed annual operating cost in US dollar for a DC with $V_{k \text {, }}^{r}$, i.e., $\mathrm{r} \in\{1,2, \ldots$,
$\left.\left|R_{k}\right|\right\}$, where $V_{k}^{r}$ is possible set of capacities of given $D C_{k}$.

$C_{j k}$ : A road distance in kilometres from $P C_{j}$ to $D C_{k}, \mathrm{j} \in \mathrm{J}, \mathrm{k} \in \mathrm{K}$.

$T_{k l}$ : A road distance in kilometres from $D C_{k}$ to $C P_{l}, \mathrm{k} \in \mathrm{K}, 1 \in \mathrm{L}$.

$\lambda$ : This is a unit cost for transferring 1 ton of maize crop for a $1 \mathrm{~km}$ distance, and the cost is in $\$$ (per km per ton).

\section{Decision variables for the model:}

$X_{j k}$ : Amount in tons flow from $P C_{j}$ to $D C_{k}$.

$Y_{k l}$ : Amount in tons flow from $D C_{k}$ to $C P_{l}$.

$Z_{k}^{r}$ : A binary location variable that will be 1 if a $D C_{k}$ is selected with a capacity $V_{k}^{r}$, and 0 otherwise. When a single capacity per $D C_{k}$ is used we ignore the superscript $r$ in $Z_{k}^{r}, V_{k}^{r}$ and $F_{k}^{r}$. Here the choice of capacity is not a decision variable but the choice of site $k$ is.

The resulting mixed integer linear programming can then be formulated as:

${ }_{X_{j k}}{ }_{\text {Min }} Y_{k l}, Z_{k} \lambda\left[\sum_{j} \sum_{k} C_{j k} X_{j k}+\sum_{k} \sum_{l} T_{k l} Y_{k l}\right]+$ $\sum_{k} F_{k} Z_{k}$

subject to

$\sum_{k} X_{j k} \leq S j, \forall \mathrm{j} \epsilon \mathrm{J}$

$\sum_{j} X_{j k}=V_{k} Z_{k,} \forall k \in K$

$\sum_{l} Y_{k l} \leq V_{k} Z_{k}, \forall k \in K$

$\sum_{k} Y_{k l}=D_{l}, \forall l \in L$

$X_{j k} \geq 0, \forall j, k ; j \in J, k \in K$

$Y_{k l} \geq 0, \forall k, l, k \in K, l \in L$

$Z_{k} \in\{0,1\}, \forall k \in K$.

Where:

- The objective function (1) minimizes the total distribution cost, e.g. transportation cost from PCs to DCs and DCs to CPs, and 
fixed annual operation costs, $F_{k}$, for DCs and the corresponding capacities $V_{k}$.

- Constraints (2) are the supply constraints (PCs' capacities), where the amount to be transported from a $P C_{j}$ to the selected DCs, must not exceed its capacity, $S_{j}$.

- Constraints (3) refer to the amount supplied from $P C_{j}$ to all selected $D C_{k}$, must not exceed the DCs' capacity $V_{k}$.

- Constraints (4) refer to the amount supplied by $D C_{k}$, to all $C P_{l}, \quad l \in L$, without exceeding $V_{k} . V_{k}$ (respectively $F_{k}$ ) are the values currently used in the current transportation network with five DCs, e.g. $D C_{1}, \ldots, D C_{5}$. The capacities $V_{k}, k \in K$, are not necessarily equal.

- Constraints (5) represent the amount to be transported from all $D C_{k}, k \in K$, to the $C P_{l}$, must meet a demand, $D_{l}$, at the $C P_{l}$.

- Constraints (6) and (7) are the nonnegativity restrictions.

- Constraints (8) are binary variables.

In the optimization results, four decisions are sought as follows:-

(a) Location decisions: Where and how many DCs to locate out of $|\mathrm{K}|$ ? The optimal decisions to be made here are the number of DCs and their physical locations (i.e., values of $Z_{k}$ or $Z_{k}^{r}$ in the case of multiple capacities).

(b) Allocation decisions: Which DCs to be served by which PCs (i.e., the pair $\left(P C_{j}\right.$ $\left.\left.; D C_{k}\right) ; j \in J ; k \in K\right)$ and which CPs are to be served by which selected DCs (i.e., the pair $\left.\left(D C_{k}: C P_{l}\right) ; k \in K ; l \in L\right)$ ? The optimal results will give the allocations of DCs to PCs and CPs to DCs simultaneously.

(c) Transportation decisions: From location and allocation decisions, what is the amount to be transported from PCs to DCs (i.e. values of $X_{j k}$ ) and DCs to CPs (i.e. values of $\left.Y_{k l}\right)$ ? The transported amounts, $X_{j k}$ and $Y_{k l}$ will be determined. Hence, direct shipment routes designing from PCs to DCs and also from DCs to CPs will be established.

(d) Capacity value decisions: What is the best capacity for each $D C_{k}$ to be selected from several possible capacities, $\left\{V_{k}^{1}, \ldots, V_{k}^{\left|R_{k}\right|}\right\}$. For the case of a single capacity per $D C_{k}$ the decision variables $Z_{k}$ will suffice.

\section{Data, Results and Discussion}

Data used in this research is taken from Tanzania official records (Sima 2015), where the three layers used in the model are PCs and CPs. Road connections to the three layers form the production, storage and distribution network. The total capacity of all four PCs, $\sum_{j=1}^{4} S_{j}$, is 532,000 tons. These data are based on annual production capacity of $2011 / 2012$. The total capacity of the five DCs, $\sum_{k=1}^{5} V_{k}$ is 178,500 tons.

The CPs are classified as 93 districts as obtained from 2004 to 2010 maize distribution data. This data was collected from the head of the disaster management unit in the Prime Minister's office, in January 2011. There are 93 CPs used in this study with total demand, $\sum_{l=1}^{93} D_{l}$, of 145,144 tons. There are five DCs each having fixed capacities, $\widehat{V_{k}}$. The DCs and PCs will be denoted in terms of their indices as shown in Table 2. The computational experiments consider the cases; 1 and 2 as explained in next paragraphs. 
Sima - Mathematical Programming Model for the Two-Level Facility Location Problem ...

Table 2: Notations for DCs and PCs

\begin{tabular}{llcl}
\hline \multicolumn{1}{c}{ DCs } & $\boldsymbol{D C} \boldsymbol{C}_{\boldsymbol{k}}$ & PCs & $\boldsymbol{P \boldsymbol { C } _ { \boldsymbol { j } }}$ \\
\hline Dar es Salaam & $D C_{1}$ & Iringa & $P C_{1}$ \\
Arusha & $D C_{2}$ & Mbeya & $P C_{2}$ \\
Dodoma & $D C_{3}$ & Rukwa & $P C_{3}$ \\
Makambako & $D C_{4}$ & Ruvuma & $P C_{4}$ \\
Shinyanga & $D C_{5}$ & & \\
\hline
\end{tabular}

There are several common inputs to be used in Cases 1 and 2. These are $|J|=4,|K|=$ 5 , and $|L|=93$. Other common inputs are the PCs' fixed capacities, $S_{j}$, distances $C_{j k}$ and $T_{k l}$. The CPs' demands, $D_{l}, l=1 ; 2, \ldots,|L|$, are given as inputs to the model in all computational experiments. Also the unit transportation cost $\lambda=\$ 0.10795$ (per km per ton) was used based on 2010 conversion rate between Tanzanian currency and US $\$$. The unit transportation cost estimated is based on NFRA (National Food Reserve Agency) maize crop transportation cost in 2010. These were available data as specified to this paper.

Generally, the research data are from four sources, which are; the Tanzania National Roads Agency (TANROADS), Ministry of Agriculture, Food Security and Cooperatives (MAFSC), National Food Reserve Agency (NFRA) and the disaster management department in the Prime minister's office.

The CPLEX software (IBM ILOG Optimization studio) was used for all the computational experiments. There is no any stopping criteria imposed; and the program will stop when it cannot enumerate any more improved solutions.

Case 1: DCs' single capacity and computational results
Here, the five DC sites were considered together with their current capacities, $R_{k}=$ $\left\{\widehat{V_{k}}\right\}$. In this case, the optimization was performed with respect to the decisions (a), (b) and (c) as listed in the previous section. The model stated by (1)-(8) was used for optimization. The purpose of this case was to see if the current network is optimal.

The optimized results are summarized in Table 3 where the first 5 columns contain some inputs to the model. The last 5 columns in Table 3 present the results obtained from optimization. For example, the variable $Z_{k}$ is used to show if the corresponding DC has been selected. The selected DCs have to be supplied from the PCs and this has been shown in column 7. The notation $P C_{j}^{S}$ in this column is used to denote the $P C_{j}$ that are the suppliers of the respective DCs. For example, it can be seen from the first entry of column 7 that $P C_{1}$ and $P C_{2}$ served the $D C_{1}$. Column 8 shows the amount of supplies received by each DC from the corresponding PCs. For example, the second entry of column 8 shows that $D C_{2}$ received 39,000 tons from $P C_{1}$.

A comparison of columns under $\widehat{V}_{k}$ and $\sum_{j} X_{j k}$, is that the full capacity of each $\mathrm{DC}$ is utilized. 
Table 3: Location allocation results for true capacity in Case 1

\begin{tabular}{cccccccccc}
\hline $\boldsymbol{D} \boldsymbol{C}_{\boldsymbol{k}}$ & $\widehat{\boldsymbol{V}}_{\boldsymbol{k}}$ & $\boldsymbol{F}_{\boldsymbol{k}}$ & $\boldsymbol{P} \boldsymbol{C}_{\boldsymbol{j}}$ & $\boldsymbol{S}_{\boldsymbol{j}}$ & $\boldsymbol{Z}_{\boldsymbol{k}}$ & $\boldsymbol{P} \boldsymbol{C}_{\boldsymbol{j}}^{\boldsymbol{s}}$ & $\sum_{j} X_{j k}$ & $\left|\boldsymbol{L}_{\boldsymbol{k}}\right|$ & $\sum_{l=1}^{\boldsymbol{L k}} \boldsymbol{Y}_{k \boldsymbol{l}}$ \\
& & & & & & & & & \\
\hline$D C_{1}$ & 52,000 & 340,340 & $P C_{1}$ & 100,000 & 1 & $P C_{2} ;$ & 22,000 & 28 & 39,361 \\
& & & & & & $P C_{1}$ & $; 30,000$ & & \\
$D C_{2}$ & 39,000 & 255,260 & $P C_{2}$ & 251,000 & 1 & $P C_{1}$ & 39,000 & 18 & 39,000 \\
$D C_{3}$ & 39,000 & 255,260 & $P C_{3}$ & 140,000 & 1 & $P C_{1}$ & 39,000 & 31 & 39,000 \\
$D C_{4}$ & 34,000 & 222,530 & $P C_{4}$ & 41,000 & 1 & $P C_{2}$ & 34,000 & 11 & 13,283 \\
$D C_{5}$ & 14,500 & 94,900 & & & 1 & $P C_{2}$ & 14,500 & 10 & 14,500 \\
\hline Total & 178,500 & $1,168,290$ & & 532,000 & & & 178,500 & 98 & 145,144 \\
\hline
\end{tabular}

The notation $L_{k}$ has been introduced to denote the set of CPs (where $\left|L_{k}\right|$ is the number of CPs) served by $D C_{k}$. Hence, the total shipment to these CPs from the $D C_{k}$ is $\sum_{l=1}^{\left|L_{k}\right|} Y_{k l}$, shown in the very last column. For example, $D C_{1}$ supplied a total of 39,361 tons to $28 \mathrm{CPs}$ which it is within its capacity. Note that, a CP can get supply from more than one DC under the so called multi-sourcing. Hence, $\sum_{k=1}^{|K|}\left|L_{k}\right| \geq|L|$, as can be seen at the last entry in column under $\left|L_{k}\right|\left(\sum_{k=1}^{|K|}\left|L_{k}\right|=\right.$ $98>|L|)$. In the results, the total demands of 145,144 tons from all the CPs are satisfied (see the total value at the last row and last column, Table 3).

With respect to the location decision, Table 3 shows that all the five DCs have been selected as shown in the column under $Z_{k}$. Also with respect to the allocation decision, it can be seen in Table 3 that all the five DCs are supplied by only $P C_{1}$ and $P C_{2}$ as shown in the column under $P C_{j}^{S}$. This clearly shows that the existing network results are different from the manually operated system since the two PCs $\left(P C_{3}\right.$ and $\left.P C_{4}\right)$ are never used. This is based on the fact that the current network has been using $P C_{3}$ and $P C_{4}$ as shown in Table 4. Data in Table 4 were prepared using data obtained from NFRA. $P C_{1}$ and $P C_{2}$ are the largest producers among the four PCs as shown in Table 5. This table shows the different annual production capacities for all the four PCs. Table 3 also shows that $P C_{1}$ is the only $\mathrm{PC}$ to supply its full capacity to the DCs.

In addition, results obtained are not the same as the data used in the current network with regard to shipments between DCs to CPs. This can be seen by comparing the results under $\left|L_{k}\right|$ in Table 3 with the data in the last column of Table 4. 
Sima - Mathematical Programming Model for the Two-Level Facility Location Problem ...

Table 4: PCs to DCs supplies from manually operated current network

\begin{tabular}{|c|c|c|c|c|c|}
\hline$D C_{k}$ & $V_{k}$ & $P C_{j}$ & PCs & $X_{j k}$ & $\mid \overrightarrow{L_{k} \mid}$ \\
\hline $\mathrm{DC}_{1}$ & 52,000 & $\mathrm{PC}_{1}$ & $\mathrm{PC}_{1} ; \mathrm{PC}_{3} ; \mathrm{PC}_{4} \mathrm{PC} 4$ & 6,$305 ; 61 ; 153$ & 26 \\
\hline $\mathrm{DC}_{2}$ & 39,000 & $\mathrm{PC}_{2}$ & $\mathrm{PC}_{3}$ & 9,867 & 16 \\
\hline $\mathrm{DC}_{3}$ & 39,000 & $\mathrm{PC}_{3}$ & $\mathrm{PC}_{3}$ & 4,009 & 12 \\
\hline $\mathrm{DC}_{4}$ & 34,000 & $\mathrm{PC}_{4}$ & $\mathrm{PC}_{3}$ & 7,523 & 9 \\
\hline $\mathrm{DC}_{5}$ & 14,500 & & & & 30 \\
\hline Total & 178,500 & & & 27,918 & 93 \\
\hline
\end{tabular}

Table 5: The summary of PCs annual maize crop total production capacity in tons

\begin{tabular}{lllllll}
\hline $\boldsymbol{P} \boldsymbol{C}_{\boldsymbol{j}}$ & \multicolumn{5}{c}{ Year } & \multicolumn{2}{c}{ Average } \\
\hline & $\mathbf{2 0 0 5 / 0 6}$ & $\mathbf{2 0 0 6 / 0 7}$ & $\mathbf{2 0 0 7 / 0 8}$ & $\mathbf{2 0 0 8 / 0 9}$ & $\mathbf{2 0 0 9 / 1 0}$ & \\
\hline $\boldsymbol{P} \boldsymbol{C}_{\mathbf{1}}$ & 412,762 & 474,270 & 384,273 & 443,905 & 393,164 & 421,675 \\
$\boldsymbol{P} \boldsymbol{C}_{\mathbf{2}}$ & 293,725 & 349,094 & 494,810 & 393,406 & 621,545 & 430,516 \\
$\boldsymbol{P} \boldsymbol{C}_{\boldsymbol{3}}$ & 270,564 & 226,524 & 351,013 & 375,732 & 372,830 & 319,333 \\
$\boldsymbol{P} \boldsymbol{C}_{\mathbf{4}}$ & 211,789 & 138,269 & 236,602 & 176,876 & 289,588 & 210,625 \\
\hline
\end{tabular}

In Case 1, the total distribution cost that includes transportation costs and DCs' annual fixed operation cost is $\$ 15,570,885.08$. This is the minimum objective value obtained from optimization after 27 seconds. In Case 1, all DCs use $\bar{V}_{k}$ as their true capacities. However, at times the demand at CPs increases and therefore replenishment is needed at DCs in order to cater for the additional demand at CPs. The capacity of $D C_{5}$ is an example of this situation as it can be seen by comparing its capacities shown in Tables 3 and 6. The replenishment is carried out from the two PCs, Songea and Sumbawanga. On the other hand, the actual capacity used may not exceed the true capacity. Therefore, the true capacity and the capacity used (actual capacity) may not be the same. Since the capacity used by $D C_{k}$ varies from year to year, the maximum actual capacity, $\widehat{V_{k}}$ used by $D C_{k}$ during 2004-2010 was taken. This actual capacity for the existing network is also considered as the manually operated existing distribution network. The program had to be re-run using $\widehat{V_{k}}$ instead of $\overline{V_{k}}$ (true capacity) and results are summarized in Table 6. Other inputs to the model (1)-(8) remained the same. Columns of Table 6 contain the same headings as in Table 3. The results were analysed with respect to the three decisions which are location, allocation and transportation.

In the location decision, all the five DCs were selected as shown in column 6 . This is due to the fact that the DC capacities are equal to total CPs' demands. The columns under $\widehat{V}_{k}$ and $\sum_{l=1}^{\left|L_{k}\right|} Y_{k l}$ have the same values.

The allocation decision in column under $P C_{j}^{s}$, shows that only two $\mathrm{PCs}, P C_{1}$ and $\mathrm{PC}_{2}$, have supplied to all the five selected DCs as in the case of Table 6 . This also clearly shows that the existing network is not optimal. The overall total network distribution cost is $\$ 13,224,626.75$ with the execution time of 23 seconds. This cost is about $15 \%$ less than the cost associated with the true capacity in Table 3 , i.e., a net saving of $\$ 2.3$ million. This reduction in cost is partly contributed by $D C_{5}$ which having the larger capacity than in Table 3, now serves more CPs, i.e. 29 CPs (see Table 6) as opposed to 10 in Table 3. 
Table 6: Location allocation results for actual capacity in Case 1

\begin{tabular}{|c|c|c|c|c|c|c|c|c|c|}
\hline$D C_{k}$ & $\widehat{V_{k}}$ & $\mathrm{~F}_{\mathrm{k}}$ & $P C_{j}$ & $\mathrm{~S}_{\mathrm{j}}$ & $\mathrm{Z}_{\mathrm{k}}$ & $P C_{j}^{S}$ & $\sum_{j} x_{j k}$ & $\mid L_{k}$ & $\sum_{l=1}^{L k} Y_{k l}$ \\
\hline $\mathrm{DC}_{1}$ & 33,190 & 217,229 & $\mathrm{PC}_{1}$ & 100,000 & 1 & $\mathrm{PC}_{1}$ & 33,190 & 26 & 33,190 \\
\hline $\mathrm{DC}_{2}$ & 38,532 & 252,192 & $\mathrm{PC}_{2}$ & 251,000 & 1 & $\mathrm{PC}_{1}$ & 38,532 & 18 & 38,532 \\
\hline $\mathrm{DC}_{3}$ & 24,650 & 161,334 & $\mathrm{PC}_{3}$ & 140,000 & 1 & $\mathrm{PC}_{1}$ & 24,650 & 13 & 24,650 \\
\hline $\mathrm{DC}_{4}$ & 9,843 & 64,422 & $\mathrm{PC}_{4}$ & 41,000 & 1 & $\mathrm{PC}_{1}$ & 24,650 & 13 & 24,650 \\
\hline $\mathrm{DC}_{5}$ & 38,929 & 254,790 & & & 1 & $\mathrm{PC}_{1} ; \mathrm{PC}_{2}$ & 3,$628 ; 6,215$ & 9 & 9,843 \\
\hline Total & 145,144 & 949,967 & & 532,000 & & & 145,144 & 95 & 145,144 \\
\hline
\end{tabular}

Case 2: DCs' multi-capacity and computational results

In this case, the main focus is given to the use of multiple capacities per DC. Unlike Case1, here the capacity of a selected DC is an optimization decision. Hence the mathematical model used in this case is re-written as follows: $\underset{X_{j k}, Z_{k}^{r}, Y_{k l},}{\operatorname{Min}} \quad \lambda\left[\sum_{j} \sum_{k} C_{j k} X_{j k}+\right.$ $\left.\sum_{k} \sum_{l} T_{k l} Y_{k l}\right]+\sum_{k} \sum_{r} F_{k}^{r} Z_{k}^{r}$ subject to

$$
\begin{gathered}
\sum_{k} X_{j k}=\sum_{k}^{(2),(5),(6) \&(7)} V_{k}^{r} Z_{k}^{r}, \forall \mathrm{k} \\
\sum_{j} Z_{k}^{r} \leq 1, \forall k \\
\sum_{l} Y_{k l} \leq \sum_{r} V_{k k}^{r} Z_{k}^{r} \forall k \\
Z_{k}^{r} \in\{0,1\}, \forall r, k
\end{gathered}
$$

Where:

- Constraints (11) refer to the amount supplied from $P C_{j}$ to all selected $D C_{k}$, that satisfy the DCs' capacity level $V_{k}^{r}$;

- Constraints (12) are now introduced to make sure that only one capacity level of selected DC is chosen. If $D C_{k}$ is selected then the constraint (12) makes sure that only one of its capacity is chosen. i.e., $\sum_{r} Z_{k}^{r}=1$. If $D C_{k}$ is not chosen, then $\sum_{r} Z_{k}^{r}=0$;

- When $D C_{k}$ is selected along with a capacity level, then constraint (13) makes sure that its $V_{k}^{r}$ for some $\mathrm{r}$ is not violated. The values of $\mathrm{r} \geq 1$, are in different ranges, some less than or equal to $\widehat{V_{k}}$ and some are more than $\overline{V_{k}}$, the existing true capacity.

- Constraints (14) are the binary values to the location variable.

The objective function (9) differs from the existing ones in literature in that the last term is modified to account for the dependence of $\mathrm{F}_{\mathrm{k}}^{\mathrm{r}}$ or $V_{k}^{r}$. The optimization of the model (9)-(14) uses the inputs data as in Case 1 except for each $D C_{k}$, which uses 14 different capacities, i.e. $R_{k}=\left\{V_{k}^{1}, V_{k}^{2}, \ldots \ldots ., V_{k}^{14}\right\}$ has been done. In the given capacities, the Case 1 capacities, $\widehat{V_{k}}$ and $\overline{V_{k}}$ are also included. Values in the set $R_{k}$ are independent of $\mathrm{k}$. The set $R_{k}, \mathrm{k}=1 ; 2, \ldots, 5$, contains the capacities that are generated randomly in $[9,843: 145,144]$. The interval from 9,843 to 145,144 was used since this range contains the minimum capacity as observed in DCs' actual capacity $\widehat{V_{k}}$, and also the maximum actual capacity used.

The optimization in this case is performed with respect to all four decisions, as done in the previous model ((a) - (d)). By optimizing the model using the original five DCs the results obtained are summarized in Table 7 , where the optimized capacity chosen are shown in brackets in the column under $V_{k}^{r}$. The results in Table 7 are self-explanatory. Table 7 shows that only four DCs are selected. 
Sima - Mathematical Programming Model for the Two-Level Facility Location Problem ...

Table 7: Location allocation results in Case 2

\begin{tabular}{cccccccccc}
\hline$D C_{k}$ & $P C_{j}$ & $S_{j}$ & $\left(Z_{k}^{r} ; r\right)$ & $V_{k}^{r}$ & $F_{k}^{r}$ & $P C_{j}^{S}$ & $\sum_{j} X_{j k}$ & $\left|L_{k}\right| \sum_{l=1}^{\left|L_{k}\right|} Y_{k l}$ \\
\hline$D C_{1}$ & $P C_{1}$ & 100,000 & $(1 ; 3)$ & $V_{1}^{3}(25,000)$ & $F_{1}^{3}$ & $P C_{1}$ & 25,000 & 20 & 25,000 \\
$D C_{2}$ & $P C_{2}$ & 251,000 & $(0 ;-)$ & - & - & - & - & - & - \\
$D C_{3}$ & $P C_{3}$ & 140,000 & $(1 ; 3)$ & $V_{3}^{3}(71,000)$ & $F_{3}^{3}$ & $P C_{1}$ & 71,000 & 39 & 71,000 \\
$D C_{4}$ & $P C_{4}$ & 41,000 & $(1 ; 13)$ & $V_{4}^{13}(16,000)$ & $F_{4}^{13}$ & $P C_{1} ; P C_{2}$ & 4,$000 ; 12,000$ & 13 & 16,000 \\
$D C_{5}$ & & $(1 ; 3)$ & $V_{5}^{3}(33,144)$ & $F_{5}^{3}$ & $P C_{2}$ & 33,144 & 24 & 33,144 \\
\hline Total & & & & & & & & & \\
\hline
\end{tabular}

NOTE: '-' This means the corresponding DC is not selected.

The optimal decisions for the capacity of DCs

are presented in column 8 under $\sum_{j} X_{j k}$ As

shown in Table 7 , the total optimal capacity of the four selected DCs is 145,144 tons which is the same as the total CPs' demand. $D C_{3}$ has the largest capacity (71,000 tons) for all the selected DCs. This is an increase of 32,000 tons from its true capacity of 39,000 tons $\left(\widehat{V}_{k}\right)$. $D C_{5}$ also needs to be increased from its true capacity of 14,500 tons to the capacity of 33,144 tons. The results obtained indicate the need for expansions for the capacities of $D C_{3}$ and $D C_{5}$.

The overall distribution cost obtained after 16 seconds is $\$ 12,660,522.80$. The total cost attained in Case 2 is the best solution for the existing maize crop distribution network in Tanzania. The cost has decreased, in comparison to Case 1, by $4.27 \%$ (actual capacity) with a net saving of $\$ 564$ thousand which is an important saving to be considered. In the case of true capacity, the saving is $18.69 \%$ which is equivalent to $\$ 2,910,000$. The saving is contributed by using many capacities that the program will select the best in each DC as compared to a single capacity as used in Case 1.

\section{Conclusion and Recommendations}

This study applied mathematical programming model to solve a two-level facility location problem for the Tanzania emergence maize distribution network where 93 CPs, 5 DCs and 4 PCs are considered. The total cost obtained in the computational results considered the three layers simultaneously. This is different from the existing system which is manually operated and has two different departments working independently. The existing distribution system has two different independent tasks carried out by specific different government departments. The first task is dealing with buying maize from PCs and transporting them for stocking in DCs which is done by the NFRA (first department). The second task is the transportation of maize from DCs to CPs which is done by the disaster management department in the Prime Minister's Office (PMO) (second department). This results into high costs due to fragmented co-ordination since the two departments operate independently and under different ministries which breeds inefficiency. The integrated coordination, as supported by this study, will reduce the costs and offer a more flexible system. Based on the discussed facts from optimization results, the study concludes the following: The use of 
optimization as a decision tool is an important aspect to be considered by the agencies in food security system and other sectors. For example, the saving of TZS 2.9 billion is a significant amount achieved through optimization. The results from this study can be applied to organize the current distribution system. In particular, Case 2 can be implemented since the existing DCs can be used together with storage facilities for the restocking of the DCs like $D C_{3}$ and $D C_{5}$. It is possible to optimize activities of food production, storage and final distribution to customers. In order to achieve this, data availability, coordinating management and the funding to the coordinating team are of great importance.

The following are recommended for further studies:

1. Extending the study to other crops especially rice which is also a common staple food. Since these products are transported to the same locations and customers, a study that involves the combination of the two products is a valuable contribution.

2. Although the study involved a mixed integer programming problem, with the exception of the binary variables, the rest were continuous variables and there possible to obtain optimal solution through available software. However, inclusion of more factors such as extension to more customer centres may require heuristic techniques.

3. The current problem considers deterministic parameters; however more realistic version considers demand as stochastic since disasters are unpredictable. Stochastic programming and other mathematical techniques may be considered as a further research area.

\section{Acknowledgements}

The author sincerely acknowledges the University of Dar es Salaam though the office of Deputy Vice Chancellor-Research for financial supporting towards writing of this paper.

\section{References}

Ahuja R, Magnanti TL and Orlin JB 1993 Network Flows: Theory, algorithms and applications. Prentice Hall, Englewood Cliffs, NJ.

Ambrosino D and Scutella MG 2005 Distribution network design: New problems and related models. Eur. J. Oper. Res. 165: 610-624.

Ameli MS, Azad N and Rastpour A 2009 Designing a supply chain network model with uncertain demands and lead times. $J$. Uncertain Syst. 3(2): 123-130.

Azad N and Davoudpour H 2013 Designing a stochastic distribution network model under risk. Int. J. Adv. Manuf. Technol. 64(23): 23-40.

Cox DW 1998 An Airlift hub-and-spoke location-routing model with time windows: Case study of the CONUS-to-Korea Airlift problem. MSc dissertation.

Elhedhli S and Goffin JL 2005 Efficient production-distribution system design. Manag. Sci. 51(7): 1151-1164.

Hindi KS, Basta T and Pienkosz K 1998 Efficient solution of a multi-commodity, two stage distribution problem with constraints on assignment of customers to distribution centres. Int. Trans. Oper. Res. 5(6): 519-527.

Klose A and Drexl A 2005 Facility location models for distribution system design. Eur. J. Oper. Res. 162: 4-29.

Lashine SH, Fattouh M and Issa A 2006 Location/allocation and routing decisions in supply chain network design. J. Model. Manag. 1(2): 173-183.

Melo MT, Nickel S and Saldanha-da-Gama F 2009 Facility location and supply chain management: a review. Eur. J. Oper. Res. 196: 401-412.

Mkenda BK and Campenhout BV 2011 International Growth Center: Estimating transaction costs in Tanzanian supply chains (Working Paper 11/0898, November). 
Sima - Mathematical Programming Model for the Two-Level Facility Location Problem ...

http:www:theigc:org/sites/default/files/esti matingtransactioncosts0:pdf.

Nagy G and Salhi S 2007 Location-routing: Issues, models and methods. Eur. J. Oper. Res. 177: 649-672.

Sahin G and Sural H 2007 A review of hierarchical facility location models. Comput. Oper. Res. 34: 2310-2331.

Sajjadi SR 2008 Integrated supply chain: Multi products location routing problem Integrated with inventory under stochastic demand. PhD thesis, Wichita State University-USA.

Sima SA 2015 Integrated stochastic distribution network design: A two-level capacitated facility location problem with application to maize crop transportation in Tanzania. PhD Thesis, University of the Witwatersrand-South Africa.
Snyder LV 2006 Facility location under uncertainty: a review. IIE Trans. 38(7): 547-564.

URT MAFSC (United Republic of Tanzania Ministry of Agriculture Food Security and Cooperatives) 2013 AGSTATS for Food Security, Volume 1: The 2011/12 Final Food Crop Production Forecast for 2012/13 Food Security, Crop Monitoring and Early Warning National Food Security Division Ministry of Agriculture Food Security and Cooperatives, Dar es Salaam. https://www.kilimo.go.tz/uploads/AGSTAT S-Fin2012-Executive_Summary-PrepADCMEW-ao210313-PUBLIC.pdf

USAID 2011 United Republic of TanzaniaFood security report, 2006. Survey and mapping of grain storage facilities in Tanzania, Tanzania country report by USAID COMPETE. 